\title{
Un État Des Lieux Général De La Protection De L'Eau En Droit International $^{1}$
}

\section{Uluslararası Hukukta Suyun Korunmasının Genel Bir Envanteri Mehtap KAYGUSUZ AKBAY*}

\begin{abstract}
Résumé
L'eau se trouve dans des conférences, instruments et jurisprudence internationaux et il existe un rapport important entre le droit international et l'eau. Cette ressource vitale bénéficie d'une protection juridique qui n'est toujours pas suffisante. L'eau, en effet, est devenue l'objet de la protection juridique d'une part à travers la protection des ressources en eau et d'autre part celle du droit à l'eau. L'objectif de la présente étude est d'aborder cette question -en mettant l'accent sur l'acquisition de l'eau d'un statut juridique en droit international, le bassin hydrographique et la notion de gestion intégrée et durable ainsi que sur la protection du droit à l'eau reconnu aux niveaux universel, régional et national-.
\end{abstract}

Mots-clés : ressources en eau, droit à l'eau, droit international.

$\ddot{O} \mathbf{z}$

Su uluslararası konferanslarda, metinlerde ve içtihatta yer almaktadır ve uluslararası hukuk ile su arasında önemli bir ilişki bulunmaktadır. Bu yaşamsal kaynak, hala yeterli olmayan hukuki bir korumadan yararlanmaktadır. Su aslında bir taraftan su kaynaklarının korunması ve diğer taraftan da su hakkının korunması yoluyla bir hukuki korumanın nesnesi haline gelmiştir. Mevcut çalışmanın amacı, bu konuyu, - suyun uluslararası hukukta hukuki bir statü elde etmesini, su havzası ve entegre ve sürdürülebilir yönetim kavramını ve ayrıca evrensel, bölgesel ve ulusal düzeylerde tanınan su hakkının korunmasını vurgulayarak-, ele almaktır.

Anahtar Kelimeler : su kaynakları, su hakkı, uluslararası hukuk.

\section{Introduction}

Objet de raréfaction, de dégradation, d'une répartition et d'une utilisation inégale, ${ }^{2}$ l'eau a trouvé sa place dans de nombreuses conférences internationales ${ }^{3}$ à côté des conférences portant directement sur l'eau. ${ }^{4}$ Certaines déclarations internationales font également référence à l'eau, comme le principe 2 de la Déclaration de Stockholm adoptée au cours de la Conférence des Nations Unies sur l'environnement en date de 1972. Elle a également apparu dans des contentieux internationaux. ${ }^{5}$ Tout cela indique le rapport entre le droit international et l'eau, qui est par ailleurs indéniable.

Il existe certes des travaux doctrinaux concernant la protection de l'eau dont une partie se concentre sur un aspect spécifique alors qu'une autre partie l'examine d'une manière globale et détaillée. Mais la présente étude vise à aborder ladite question dans les grandes lignes sans entrer dans les détails et ainsi à faire un état des lieux de la protection de l'eau en droit international. En effet, cette étude peut être considérée comme une revue générale dudit thème.

L'une des questions principales est celle de savoir comment cette ressource vitale est devenue l'objet d'une protection internationale. Cette question peut être abordée sous le

\footnotetext{
* Dr., İstanbul Üniversitesi, Siyasal Bilgiler Fakültesi, mehtap.kaygusuz@istanbul.edu.tr

${ }^{1}$ La présente étude est une version développée et mise à jour de la contribution présentée (sous le titre «Aspects normatifs») à la "Journée d'études du Centre de droit international» intitulée "L'eau en droit, l'eau et le droit : problèmes et enjeux actuels » qui a eu lieu le 9 décembre 2013 au sein de l'Université Lyon 3.

${ }^{2}$ Lavieille, 2010, pp. 226-228.

${ }^{3}$ Par exemple la Conférence de Stockholm de 1972, la Conférence de Rio de 1992 ou la Conférence internationale sur la population et le développement de 1994.

${ }^{4}$ Voir la Conférence des Nations Unies sur l'eau de Mar del Plata de 1977, la Conférence internationale sur l'eau et l'environnement de 1992 ou la Conférence internationale sur l'eau et le développement de 1998.

${ }^{5}$ Par exemple l'affaire du lac Lanoux de 1957 ; l'affaire relative au projet Gabč́kovo-Nagymaros de 1997 ou celle relative à des Usines de pâtes à papier sur le fleuve Uruguay de 2010.
} 
prisme de la protection d'une part des ressources en eau et d'autre part du droit à l'eau. Cela constitue le cadre du présent article et une démarche donnant lieu de dresser un bilan général autour de la question mentionnée ainsi que de constater l'état actuel de la protection de l'eau en droit international.

Le premier aspect, - la protection des ressources en eau-, semble appeler d'une part l'examen de l'acquisition de l'eau d'un statut juridique permettant d'évoquer un bref historique et d'indiquer des instruments importants en la matière. D'autre part, en considérant l'état actuel de la protection des ressources en eau, cet aspect conduit à rappeler la nécessité d'une protection plus appropriée. ${ }^{6}$

Quant au second aspect, -le droit à l'eau-, il a un rapport important avec la protection de l'eau, de telle sorte que deux protections entretiennent un rapport de " complémentarité » plutôt que celui de "concurrence ", comme le dit le Professeur Dupuy. ${ }^{7}$ Étant donné que le droit à l'eau est reconnu aux plans universel, régional ainsi que national, il conviendra de se pencher sur ce thème en prenant en compte ces trois niveaux.

\section{La Protection Juridique Des Ressources En Eau}

Les ressources en eau, étant apparues en tant que concept au $\mathrm{XX}^{\mathrm{e}}$ siècle, ${ }^{8}$ bénéficient à l'heure actuelle d'une protection étant insuffisante. ${ }^{9}$ Avant d'examiner la nécessaire protection desdites ressources portant sur une approche plus appropriée, ${ }^{10}$ il semble important de démontrer que l'eau a acquis un statut juridique en droit international.

\section{L'Acquisition D'Un Statut Juridique En Droit International}

Pendant longtemps, ce sont les fleuves qui étaient l'objet des règles internationales en tant que voies de navigation, mais pas « l'eau en tant que telle $» .{ }^{11}$

$\mathrm{Au}$ niveau universel, plusieurs travaux internationaux témoignent de la prise en compte de l'eau elle-même depuis quelques années déjà. C'est en 1971 que la Commission du droit international (CDI), organe subsidiaire des Nations Unies, a débuté ses travaux sur les « utilisations des cours d'eau à des fins autres que la navigation ». ${ }^{12}$ Ces travaux ont été suivis de l'adoption des deux traités : d'abord la Convention sur la protection et l'utilisation des cours d'eau transfrontières et des lacs internationaux de 1992 (dite Convention d'Helsinki) ${ }^{13}$ et ensuite, la Convention sur le droit relatif aux utilisations des cours d'eau internationaux à des fins autres que la navigation de 1997 (dite Convention de New York). ${ }^{14}$ La CDI a, en outre, adopté en 2008 le projet d'articles sur le droit des aquifères transfrontières. ${ }^{15}$ À ces instruments, il faut ajouter d'autres tels que les Règles d'Helsinki sur l'utilisation des eaux des fleuves internationaux de 1966, les Règles de Séoul sur les eaux souterraines internationales

\footnotetext{
${ }^{6}$ Voir l'article 7 du Projet d'articles sur le droit des aquifères transfrontières et Doumbé-Billé, 1994, p. 203.

${ }^{7}$ « [I]l n'y a pas concurrence mais complémentarité entre droit de et droit à l'eau. Le premier appartient aux États; le second à l'individu. Mais, on l'a dit assez, le premier s'exerce au bénéfice du second ». DUPUY, 2006, p. 12.

${ }^{8}$ Paquerot, 2011, p. 72.

${ }^{9}$ Voir Doumbé-Billé, 1994, p. 202.

${ }^{10}$ Voir l'article 7 du Projet d'articles sur le droit des aquifères transfrontières et Doumbé-Billé, 1994, p. 203.

11 Paquerot, 2011, pp. 73-74.

12 AGNU, Rés. 2669 (XXV), 8 décembre 1970 et A/CN.4/244/Rev.1.

${ }^{13}$ Cette Convention est entrée en vigueur en 1996. Avec l'adoption des amendements aux articles 25 et 26 de la Convention d'Helsinki, cette dernière est devenue une convention ouverte à la signature de tous les États membres des Nations Unies (Pour l'amendement voir ECE/MP.WAT/14, 12 janvier 2004). En la matière voir Arcari, 2012, pp. 350 et s.

${ }^{14}$ La Convention de New York est entrée en vigueur le 17 août 2014.

${ }^{15}$ Annuaire de la Commission du droit international, 2008, Vol. II (Deuxième partie), pp. 21 et $\mathrm{s}$.
} 
de 1986, les Règles de Berlin sur les ressources en eau de 2004 adoptées par l'Association de droit international.

Le droit international a en effet connu une évolution en matière de protection des ressources en eau : ${ }^{16}$ alors qu'il ne prenait en compte qu'accessoirement la protection des fleuves contre la pollution, il a subi un changement grâce à l'adoption des traités sur la protection des eaux fluviales. ${ }^{17}$ En d'autres termes, l'entrée sur la scène internationale des traités contenant des dispositions relatives à la protection de l'environnement aquatique à partir de la deuxième moitié du $19^{\mathrm{e}}$ siècle a été suivie par la multiplication des traités relatifs à la pollution des eaux à partir de la seconde moitié du $20^{\mathrm{e}}$ siècle. $^{18}$

L'eau dispose d'un statut en droit international qui est susceptible de se modifier. ${ }^{19}$ Considérée généralement comme une ressource naturelle à laquelle s'appliquent les principes de souveraineté ${ }^{20}$ et de liberté des échanges, l'eau est désormais envisagée sous différentes approches soit comme une ressource économique, soit comme un bien commun mondial ou un patrimoine commun de l'humanité. ${ }^{21}$

Si l'eau est devenue un objet de la protection juridique, cette protection demeure néanmoins faible. Une des raisons de cet état actuel est la résistance des États en la matière, comme le montrent la non-entrée en vigueur de la Convention de New York pendant très longtemps (comme il a été déjà précisé, elle a été adoptée en 1997 et n'est entrée en vigueur qu'en 2014) et la contestation relative aux Règles de Berlin de 2004. ${ }^{22}$ Pour pouvoir dépasser cette résistance, le projet d'articles sur le droit des aquifères transfrontières adopté en 2008 par la CDI met l'accent sur la souveraineté contrairement à la Convention de New York. ${ }^{23}$ En outre, en dépit de l'existence d'un très grand nombre d'instruments juridiques, ${ }^{24}$ certains bassins fluviaux internationaux ne font toujours pas l'objet d'un instrument conventionnel. ${ }^{25}$ À cela s'ajoutent l'état sectoriel des instruments juridiques, ${ }^{26}$ l'état fragmenté du droit international qui s'applique à $l^{\prime} \mathrm{eau}^{27}$ et la situation inadaptée du système international. ${ }^{28} \mathrm{Il}$ est donc nécessaire d'assurer une protection plus appropriée ${ }^{29}$ de l'eau, car il s'agit de la mise en cause " non seulement [de] la vie, mais [de] la survie même de la planète » par la dégradation et le multiple usage des eaux douces. ${ }^{30}$

${ }^{16}$ Concernant l'évolution voir Brown Weiss, 2009, pp. 231 et s. ; Coulée, 2011, pp. 27 et s. ; Arcari, 2012, pp. 350 et s. ; Boisson de Chazournes et Tignino, 2013 et Sohnle, 2011, pp. 306 et s.

${ }^{17}$ Ngyuen, Daillier, Forteau et Pellet, 2009, p. 1461.

18 Sohnle, 2011, pp. 306-307.

${ }^{19}$ Boisson de Chazournes et Tignino, 2013.

${ }^{20}$ Voir par exemple AGNU, Rés. 1803 (XVII), 14 décembre 1962.

${ }^{21}$ Cardenas, 2007, pp. 3 et s. ; Paquerot, 2002, pp. 214 et s. ; Boisson de Chazournes et Tignino, 2013 , § 132. Voir par exemple Agenda 21, A/CONF.151/26/Rev.1, Chapitre 18, § 18. 8.

22 Paquerot, 2011, p. 83 et Arcari, 2012, p. 357.

${ }^{23}$ Sohnle, 2011, p. 314.

${ }^{24}$ Selon l'Organisation des Nations Unies pour l'alimentation et l'agriculture (FAO), il existe plus de 3600 traités relatifs aux ressources en eau. Ces chiffres comprennent cependant les traités de navigations et de démarcation. Coulée, 2011, pp. 23-24.

25 Arcari, 2012, pp. 349-350.

${ }^{26}$ Coulée, 2011, p. 24 et Sohnle, 2002, pp. 97 et s.

${ }^{27}$ Cuq, 2013, pp. 21 et s.

${ }^{28}$ Paquerot, 2002, p. 196.

${ }^{29}$ Voir l'article 7 du Projet d'articles sur le droit des aquifères transfrontières et également Doumbé-Billé, 1994, p. 203.

${ }^{30}$ Doumbé-Billé, 1994, pp. 197-198. 


\section{La Nécessité D’Une Protection Plus Appropriée}

Les liens profonds entre les problèmes écologiques et la question des ressources en eau nécessitent une protection fondée sur une approche plus appropriée, ${ }^{31}$ plus compatible avec l'eau qui, comme le précise le principe 12 de la Charte européenne de l'eau de 1968, « n'a pas de frontière ». Une telle protection exige la prise en compte du bassin hydrographique d'une part et de la notion de gestion intégrée et durable d'autre part. ${ }^{32}$

\section{La prise en compte du bassin hydrographique}

La notion de bassin hydrographique inclut «tout le territoire sur lequel des goûtes d'eau incorporées au sol ruissellent vers un même cours d'eau $\gg .{ }^{33}$ La référence explicite ou implicite à ladite notion peut être considérée comme un développement vers une protection globale se fondant sur une approche intégrée qui prend en compte «l'ensemble du bassin hydrographique $» .{ }^{34}$ Dans cette lignée, l'unité hydrographique des cours d'eau est reconnue par la jurisprudence internationale ${ }^{35}$ et par des instruments internationaux, tels que les Règles d'Helsinki de 1966 ou encore la Convention d'Helsinki de 1992 (art. 2 6, art. $9 \S \S 1$ et 5). ${ }^{36}$

Par contre, la Convention de New York étant une convention à vocation universelle, n'emploie pas la notion de "bassin hydrographique », mais définit les cours d'eau comme suit: "L'expression "cours d'eau" s'entend d'un système d'eaux de surface et d'eaux souterraines constituant, du fait de leurs relations physiques, un ensemble unitaire et aboutissant normalement à un point d'arrivée commun » (art.2, §a). Ainsi, l'article cité utilise la notion de «système de cours d'eau international » qui « compren[d] les eaux superficielles et souterraines, mais pas leur support terrestre $\gg .{ }^{37}$ Par conséquent, la Convention de New York adopte une approche plus restrictive en la matière. ${ }^{38}$

\section{La notion de gestion intégrée et durable}

L'« approche intégrée » a été encouragée par l'Agenda 21 selon lequel

[p]our mettre en valeur et gérer les ressources en eau, il convient d'adopter une approche intégrée qui tienne compte des besoins à long terme comme des besoins immédiats. Tous les facteurs, qu'ils soient écologiques, économiques ou sociaux, devront donc être pris en considération dans l'optique d'un développement durable. ${ }^{39}$

D'après l'Agenda 21, la «gestion intégrée des ressources en eau » résulte de l'idée selon laquelle, d'une part, l'eau est « partie intégrante de l'écosystème » et d'autre part, elle est non seulement une ressource naturelle mais également un bien à la fois social et économique dont l'affectation est déterminée par sa quantité et sa qualité. ${ }^{40}$ La protection de cette ressource naturelle doit d'un côté prendre en compte le fonctionnement des écosystèmes

${ }^{31}$ Ibid., p. 203. Voir l'article 8 de la Convention de New York.

${ }^{32}$ Voir Boisson de Chazournes et Tignino, 2013, §86 et Doumbé-Billé, 1994, p. 200.

${ }^{33}$ Sohnle, 2011, p. 309. Voir également Paquerot, 2002, pp. 206 et s.

${ }^{34}$ Boisson de Chazournes et Tignino, 2013, §86.

Il est à noter que selon le PNUE, il existe 263 bassins fluviaux internationaux. (UNEP, 2002, p. 1). Selon les Nations Unies, " sur les 263 bassins internationaux existants, plus de 150 - auxquels il faut ajouter des systèmes aquifères transfrontières - ne sont l'objet d'aucun cadre de gestion en coopération, de quelque type que ce soit ». A/64/692, 4 mars 2010, «Eau, paix et sécurité : la coopération dans le domaine de la gestion des eaux transfrontières », note du Secrétariat, $\S 25$.

${ }^{35}$ Par exemple, CPJI, Affaire relative à la juridiction territoriale de la Commission internationale de l'Oder, 10 septembre 1929, Série A, n²3. Voir Sohnle, 2011, p. 308.

36 Paquerot, 2011, p. 74.

${ }^{37}$ Sohnle, 2011, p. 309; Paquerot, 2002, pp. 206 et s.

${ }^{38} \mathrm{Idem}$.

${ }^{39}$ Agenda 21, § 18.16. Voir Boisson de Chazournes et Tignino, 2013, $\S 86$.

${ }^{40}$ Agenda 21, § 18.8 . 
aquatiques et la pérennité de la ressource et d'autre côté viser à satisfaire les besoins en eau aux fins des activités humaines. ${ }^{41}$

Selon le Protocole sur l'eau et la santé à la Convention d'Helsinki la «démarche intégrée devrait s'appliquer » non seulement à une partie du bassin hydrographique mais à son ensemble. ${ }^{42}$ L'affaire Fundación Chadileuvú c. Estado Nacional Argentino y Provincia de Mendoza s'inscrit dans cette lignée en conduisant le Tribunal Latinoamericano del Agua à faire remarquer l'absence de plan relatif à la gestion intégrée du bassin hydrographique en cause et à attirer l'attention sur le fait qu'à cause dudit manque les habitants du bassin ne pouvaient utiliser de l'eau d'une manière équitable. ${ }^{43}$

L'article $5, \S \mathrm{j}$ du Protocole sur l'eau et la santé prévoit également que :

[1] es ressources en eau devraient être gérées, dans toute la mesure possible, d'une façon intégrée au niveau des bassins hydrographiques, afin de lier, d'une part, le développement social et économique à la protection des écosystèmes naturels, et, d'autre part, la gestion des ressources en eau à des mesures réglementaires concernant d'autres secteurs de l'environnement. (C'est nous qui soulignons).

Les exemples cités indiquent des liens entre gestion durable ${ }^{44}$ et gestion intégrée. ${ }^{45}$

Le document final «L'avenir que nous voulons » adopté au cours de la Conférence des Nations Unies sur le développement durable de 2012 (Rio+20) affirme la nécessité d'une "gestion intégrée et durable des ressources naturelles et des écosystèmes » permettant notamment le développement économique, social et humain, tout en soulignant que cette gestion doit prendre en compte «la protection, la régénération, la reconstitution et la résistance des écosystèmes face aux défis existants et nouveaux $\gg{ }^{46}$ Le Programme des Nations Unies pour l'environnement (PNUE), quant à lui, reconnait, dans l'une de ses décisions datée 2013, que la promotion de la gestion durable nécessite d'une part la cohérence des directives relatives à la qualité de l'eau pour les écosystèmes et d'autre part leur intégration dans les directives existantes sur l'eau. ${ }^{47} \mathrm{La}$ durabilité doit en effet être sousentendue lorsqu'il s'agit du droit de l'eau. ${ }^{48}$ Le fait qu'aujourd'hui plusieurs pays souffrent de la pénurie d'eau ${ }^{49}$ nous rappelle encore une fois l'importance de la durabilité en matière d'eau. Dans l'affaire relative à des Usines de pâte à papier sur le fleuve Uruguay, la Cour internationale de Justice (CIJ) a eu l'occasion de se prononcer sur l'impératif d'équilibre entre l'utilisation et la protection des eaux du fleuve répondant à l'objectif de développement durable. ${ }^{50}$ Il n'est pas inutile de noter que la durabilité peut nécessiter de mettre fin à une activité économique, comme l'illustre la décision du Tribunal Latinoamericano des Agua

\footnotetext{
${ }^{41}$ Idem.

${ }^{42}$ L'article 5, § j du Protocole sur l'eau et la santé de1999. Voir Boisson de Chazournes et Tignino, $2013, \S 86$.

${ }^{43}$ Tribunal Latinoamericano des Agua, Fundación Chadileuvú c. Estado Nacional Argentino y Provincia de Mendoza, 5 novembre 2012. Cité par WASH United and Waterlex, 2014, p. 283.

${ }^{44} \mathrm{La}$ notion de gestion durable se trouve dans certains instruments juridiques internationaux tels que la Convention de New York (art.24), la Convention d'Helsinki (art.3), les Règles de Berlin (art.7), ainsi que dans la jurisprudence, telle que l'affaire relative à des Usines de pâte à papier sur le fleuve Uruguay (§ 184).

${ }^{45}$ Concernant le principe de gestion intégrée voir Sohnle, 2002, p. 182; Conseil de l’Union européenne, «Protection des ressources en eau et gestion durable intégrée de l'eau dans l'Union européenne et au-delà Conclusions du Conseil- », 3013 ${ }^{\text {ème }}$ session du Conseil Environnement, 21 juin 2011.

${ }^{46}$ L'avenir que nous voulons, A/RES/66/288, 27 juillet 2012, § 4.

47 PNUE, Décision 27/3: Directives internationales sur la qualité de l'eau pour les écosystèmes, UNEP/GC.27/17, $27^{\text {ème }}$ session du Conseil d'administration/ Forum ministériel mondial sur l'environnement, 18-22 février 2013 .

${ }^{48}$ Association du droit international, Règles de Berlin, commentaire de l'article 7.

49 «À l'heure actuelle, quasiment tous les pays d'une ceinture située entre 10 degrés et 40 degrés environ au nord, du Mexique à la Chine et à l'Europe du Sud, sont touchés par la pénurie d'eau, comme l'Australie, l'Amérique du Sud occidentale et l'Afrique australe dans l'hémisphère Sud ». UNESCO, 2018, p. 17.

${ }^{50} \mathrm{CIJ}$, Affaire relative à des Usines de pâte à papier sur le fleuve Uruguay, § 177 ; Kohen, 2011, p. 107.
} 
relative à l'affaire Frente Amplio Opositor a Minera San Xavier c./ Minera San Xavier SA de $C V$ y Otros. Dans cette affaire le Tribunal a recommandé d'arrêter les activités minières ainsi que de respecter des normes non seulement nationales mais également internationales relatives à la protection de l'aquifère - en l'espèce celui de San Luis Potosí-. ${ }^{51}$

S'agissant des écosystèmes, dans l'affaire relative à des Usines de pâte à papier, la Cour de La Haye indiquait l'importance de la vigilance et de la prévention en matière de préservation de l'équilibre écologique car les conséquences des activités humaines risquent d'être néfastes pour d'autres composantes de l'écosystème. ${ }^{52} \mathrm{Ce}$ risque de détérioration de l'écosystème était par ailleurs l'un des arguments défendus devant la CIJ par le Nicaragua concernant la construction d'une route au Costa Rica le long du fleuve San Juan. ${ }^{53}$

La gestion intégrée nécessite la prise en considération de «l'écosystème aquatique intégré » fondé sur les règles de fonctionnement non seulement du milieu naturel mais également des activités humaines. ${ }^{54}$ En matière de gestion intégrée la professeure Coulée attire l'attention sur l'exigence de deux conditions : d'abord la conciliation de "la réalité physique de l'eau avec les constructions juridiques » et ensuite, la prise en compte des préoccupations d'intérêts communs. ${ }^{55}$

Si la gestion intégrée et durable de l'eau est considérée nécessaire, elle n'est néanmoins pas une chose aisée, car, d'une part, elle s'appréhende par différentes branches du droit international (comme le droit international économique, le droit international du commerce ou le droit international de l'environnement), et d'autre part elle présente un usage multiple. ${ }^{56}$ Certaines difficultés relatives à la gestion durable ont déjà été mises en évidence par exemple par la mise en œuvre de l'Accord de coopération pour la mise en valeur durable du bassin Mékong de 1995 et de l'Accord de Farakka. ${ }^{57}$ Au-delà de la pratique, à l'heure actuelle les instruments internationaux ne prennent pas véritablement en compte le cycle hydrologique, la protection de l'écosystème du bassin, toutes les formes de l'eau et tous les usages de l'eau. ${ }^{58}$

Concernant les usages de l'eau, la Convention de New York de 1997 prévoit qu' « [e]n cas de conflit entre des utilisations d'un cours d'eau international, [...], une attention spéciale étant accordée à la satisfaction des besoins humains essentiels » (art. 10, § 2) ou encore l'article $5, \S 2$ du projet d'articles sur le droit des aquifères transfrontières mentionne « des besoins humains vitaux $»$, ceux qui nous amènent au droit à l'eau. ${ }^{59}$

\section{La Protection Du Droit À L'Eau}

En 2000, le Secrétaire général des Nations Unies, Kofi Annan, énonçait que «[1]e problème le plus grave qui se pos[ait] dans l'immédiat [était] que plus d'un milliard de

\footnotetext{
51 Tribunal Latinoamericano des Agua, Frente Amplio Opositor a Minera San Xavier c./ Minera San Xavier SA de CV y Otros, 11 octobre 2007. Cité par WASH United and Waterlex, 2014, p. 285.

${ }_{52} \mathrm{CIJ}$, Affaire relative à des Usines de pâte à papier sur le fleuve Uruguay, § 188.

${ }^{53}$ CIJ, Construction d'une route au Costa Rica le long du fleuve San Juan (Nicaragua c. Costa Rica), Requête introductive d'instance du 22 décembre 2011, § 4. Voir également CIJ, Affaire relative à des Usines de pâte à papier sur le fleuve Uruguay, Requête introductive d'instance du 4 mai 2006, § 5.

${ }^{54}$ Sohnle, 2002, p. 178.

${ }^{55}$ Coulée, 2011, pp. 27, 28, 30.

${ }^{56}$ Paquerot, 2011, p. 83 ; Dupuy, 2006, p. 5 et Cuq, 2013, p. 17.

57 À ce propos voir Lawson, 2013, pp. 323 et s.

${ }^{58}$ Sohnle, 2002, p. 35 ; Brown Weiss, 2009, pp. 221-222; Sohnle, 2011, p. 310 et Cuq, 2013, pp. 31-32, 83.

${ }^{59}$ Voir Boisson de Chazournes et Tignino, 2013, § 157 ; Dupuy, 2006, pp. 4 et 2 ; Dan Tarlock, A. (2017), § $11: 17$.
} 
personnes n'[avait] pas accès à l'eau potable » en ajoutant que « la moitié de l'humanité ne dispos[ait] pas de moyens d'assainissement adéquats $\gg .{ }^{60}$

La question du droit à l'eau est une question vitale. Et la protection dudit droit a des liens avec la protection de l'eau elle-même, étant donné que ce droit englobe, entre autres, la qualité de l'eau. ${ }^{61} \mathrm{D}$ 'après l'Observation générale $\mathrm{n}^{\circ} 15$ adoptée en 2002 par le Comité des droits économiques, sociaux et culturels, cette qualité renvoie à la salubrité de l'eau et au fait que l'eau doive avoir une couleur, une odeur et un goût « acceptables ». ${ }^{62}$ La qualité de l'eau concerne sa protection contre la contamination (le déversement des eaux usés et des déchets, l'infiltration de polluants dans les eaux souterrains etc.). ${ }^{63}$ Ainsi, les États doivent prendre les mesures nécessaires afin d'empêcher la pollution, la contamination de $1^{\prime}$ 'eau ${ }^{64}$ et de garantir que l'eau ait une qualité acceptable. ${ }^{65}$ Le droit à l'eau ayant donc une dimension concernant la qualité de l'eau, a été reconnu à la fois au niveau universel et aux niveaux régional et national.

\section{La Reconnaissance Du Droit À L'Eau Au Niveau Universel}

Que faut-il entendre par «droit à l'eau»? Selon le Comité des droits économiques, sociaux et culturels, il « consiste en un approvisionnement suffisant, physiquement accessible et à un coût abordable, d'une eau salubre et de qualité acceptable pour les usages personnels et domestiques de chacun $\gg{ }^{66}$

Certains droits entretiennent des liens étroits avec le droit à l'eau. ${ }^{67} \mathrm{Il}$ en est ainsi pour le droit à la santé, le droit à un niveau de vie suffisant, le droit à l'environnement, le droit des peuples autochtones, le droit à l'alimentation, le droit à un logement, le droit au développement ou encore le droit de propriété. ${ }^{68}$ En conséquence, dans le cadre des instruments internationaux reconnaissant lesdits droits, il peut être considéré que le droit à l'eau est consacré de façon implicite. ${ }^{69}$

Au-delà de la consécration implicite, le droit à l'eau trouve sa place dans certains instruments internationaux. Dans cette lignée, certains instruments reconnaissent le droit à l'eau mais cela uniquement pour des catégories précises telles que les prisonniers de guerre, les internés, les femmes, les enfants, les handicapés, les peuples autochtones. ${ }^{70}$ Comme le

${ }^{60}$ Rapport du Secrétaire général, Nous, les peuples : le rôle des Nations Unies au XXIe siècle, 2000, A/54/2000, p. 48, § 278 ; Lavieille, 2010, pp. 226 et 227.

${ }^{61}$ Selon le Comité des droits économiques, sociaux et culturels, les facteurs pertinents de la notion d'approvisionnement en eau adéquat sont la disponibilité, la qualité et l'accessibilité y compris l'accessibilité physique, l'accessibilité économique, la non-discrimination et l'accessibilité de l'information. L'Observation générale $\mathrm{n}^{\circ}$ 15, 2002. Voir Brown Weiss, 2009, p. 311.

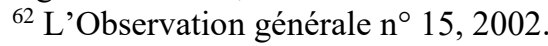

${ }^{63}$ WASH United and Waterlex, 2014, p. 30.

${ }^{64}$ Voir Tribunal Latinoamericano del Agua, Comunidades Indígenas del Cantón de Sisimitepet y Pushtan des Municipio de Nahuizalco c/ Presidencia de la República de El Salvador y Otros, 12 septembre 2008. Cité par WASH United and Waterlex, 2014, pp. 292-293.

${ }^{65}$ Voir CIADH, Xákmok Kásek Indigenous Community v Paraguay, 24 August 2010, § 196. Exemple cité par WASH United and Waterlex, 2014, p. 269.

${ }^{66}$ Comité des droits économiques, sociaux et culturels, Rapport sur les vingt-huitième et vingt-neuvième sessions (29 avril-17 mai 2002, 11-29 avril 2002), Annexe IV, Observation générale $n^{\circ} 15$ (2002), Le droit à l'eau (art.11 et 12 du Pacte), p. 132.

${ }^{67} \mathrm{~A} / \mathrm{RES} / 54 / 175$, 15 février 2000, § 12, a) ; Rapport de la Conférence internationale sur la population et le développement, Le Caire, 5-13 septembre 1994, A/CONF.171/13/Rev.1, Principe 2; Smets, 2011, pp. 357-358.

${ }^{68}$ Bluemel, 2004, p. 1005 ; Sohnle, 2002, pp. 439-440 ; Brown Weiss, 2009, pp. 309 et s.; Smets, 2002a, pp. $23-$ 24 ; Coulée, 2011, p. 34. ; Smets, 2011, p. 350 ; Pellet, 2011, p. 398 ; Caflisch, 2011, p. 390.

${ }^{69}$ Voir le commentaire de l'article 17 des Règles de Berlin ; Dupuy, 2006, pp. 3 et s. ; Smets, 2002a, pp. 24 et s. ; Caflisch, 2011, pp. 388 et s. ; Coulée, 2011, pp. 33 et s.

${ }^{70}$ Les articles 20, 26, 29 et 46 de la Convention de Genève relative au traitement des prisonniers de guerre ; les articles 85,89 et 127 de la Convention de Genève relative à la protection des personnes civiles en temps de 
confirme l'Observation générale $\mathrm{n}^{\circ} 15$ du Comité des droits économiques, sociaux et culturels, certaines catégories d'individus méritent une attention particulière, car elles «ont traditionnellement des difficultés à exercer» $»$ e droit à l'eau. ${ }^{71}$ Ainsi, il est tout à fait compréhensible et nécessaire que les instruments qui visent à protéger les catégories vulnérables reconnaissent le droit à l'eau. Mais le champ d'application de ces instruments reste limité auxdites catégories.

Pour que le droit d'accès à l'eau soit annoncé explicitement d'une manière générale, il fallait attendre la Conférence de Mar del Plata de $1977 .{ }^{72}$ Ensuite, avec la résolution 35/18 du 10 novembre 1980, l'Assemblé générale des Nations Unies (AGNU) a proclamé la Décennie internationale de l'eau potable et de l'assainissement. L'Agenda 21 a également souligné l'importance de l'approvisionnement en eau potable et l'assainissement. ${ }^{73}$ Mais le document significatif en la matière est l'Observation générale $\mathrm{n}^{\circ} 15$ mentionnée ci-dessus par laquelle le droit à l'eau a été reconnu en tant que droit de l'homme. Elle précise, d'une part, que « [1]e droit à l'eau fait clairement partie des garanties fondamentales pour assurer un niveau de vie suffisant » prévu au paragraphe 1 de l'article 11 du Pacte international relatif aux droits économiques, sociaux et culturels (PIDESC), et, d'autre part, que ce droit est « inextricablement lié au droit au meilleur état de santé » prévu au paragraphe 1 de l'article 12 du PIDESC. ${ }^{74}$ Si l'Observation générale $\mathrm{n}^{\circ} 15$ admet « les contraintes dues à la limitation des ressources disponibles », elle énumère pourtant trois types d'obligations spécifiques des États en matière du droit à l'eau, à savoir le respect, la protection et la mise en œuvre. ${ }^{75}$ L'Observation générale indique ainsi le rôle de l'État dans la réalisation de ce droit. Dans ce sens, la Cour suprême mexicaine a confirmé que l'interprétation (prenant en compte les normes internationales) de la Constitution mexicaine exige que

l’État à (faire) respecter et (faire) garantir le droit fondamental à l'eau, mais aussi à établir une stratégie pour permettre un accès et une utilisation équitable de l'eau potable dans le cadre d'un développement durable de l'exploitation des ressources en eau. ${ }^{76}$

La décision du Tribunal Lationoamercano del Agua va dans la même lignée : il rappelle que ce sont les États qui doivent protéger la pleine jouissance du droit à l'eau. ${ }^{77}$

Certes l'Observation générale $\mathrm{n}^{\circ} 15 \mathrm{n}^{\prime}$ 'est pas juridiquement contraignante pour les États, mais cela n'empêche pas que des juridictions s'y réfèrent. Par exemple, dans l'affaire Xákmok Kásek Indigenous Community v. Paraguay, la Cour interaméricaine des droits de l'homme a fait référence à l'Observation générale lorsqu'elle s'est prononcée sur la minimum quantité d'eau par jour. ${ }^{78}$ L'importance de l'Observation générale résulte en outre de

guerre; le paragraphe 2 de l'article 14 de la Convention sur l'élimination de toutes les formes de discrimination à l'égard des femmes de 1979; l'article 24-2-c de la Convention relative aux droits de l'enfant de 1989; 1'article 28 de la Convention relative aux droits des personnes handicapées, de 2006 ; les articles 25,26 et 32 de la Déclaration des Nations Unies sur les droits des peuples autochtones, A/RES/61/295, 13 septembre 2007 ; l'article 20 des Règles de Berlin ; l'article 15 de la Convention n ${ }^{\circ} 169$ de l'OIT relative aux peuples indigènes et tribaux. Voir Dupuy, 2006, pp.2 et 6 ; Boisson de Chazournes et Tignino, 2013, $\S \S 164$ et s.; Smets, 2007 , p. 30 ; Smets, 2011, p. 364 ; Brown Weiss, 2009, pp. 341 et s.

${ }^{71}$ L'Observation générale $n^{\circ} 15, \S 16$.

${ }^{72}$ Coulée, 2011, p. 34 et note 106 ; Dupuy, 2006, p. 2. ; Boisson de Chazournes et Tignino, 2013, § 160.

${ }^{73}$ Agenda 21, préc., Chapitre 18, 18.47.

${ }^{74}$ L'Observation générale ${ }^{\circ} 15$, pp. 132-133.

75 Ibid., pp. 139 et s.

${ }^{76}$ Bernaud, 2017, p. 335.

77 Tribunal Latinoamericano del Agua, Fundación Chadileuvú c. Estado Nacional Argentino y Provincia de Mendoza, 5 novembre 2012. Cité par WASH United and Waterlex, 2014, p. 283.

${ }^{78}$ CIADH, Xákmok Kásek Indigenous Community v Paraguay, 24 August 2010, § 195, note 214. Voir WASH United and Waterlex, 2014, p. 269. Voir également CIADH, Affaire Yakye Axa Indigenous Community c. Paraguay, 17 juin 2005, § 167, note 206. 
l'introduction du droit à l'eau en tant que droit impliqué dans les droits de l'homme prévus par le PIDESC dont le respect par les États est contrôlé au niveau international. ${ }^{79}$ De plus, depuis l'entrée en vigueur le 5 mai 2013 du Protocole facultatif au PIDESC, les droits prévus par le Pacte sont justiciables. ${ }^{80}$

Suite à ladite Observation, l'AGNU a adopté, en juillet 2010, une résolution par laquelle elle a reconnu le droit à l'eau potable et à l'assainissement en tant que droit de l'homme en soulignant que ce droit est fondamental pour l'exercice des droits de l'homme. ${ }^{81}$ L'adoption de cette résolution a cependant témoigné à l'abstention d'un grand nombre d'États (122 votes pour et 41 votes d'abstention) et la formulation de la résolution relative à sa portée et son caractère demeure ambiguë selon certains auteurs. ${ }^{82}$ Ladite résolution a été suivie par celles du Conseil des droits de l'homme sur les droits de l'homme et l'accès à l'eau potable et à l'assainissement de même que sur le droit fondamental à l'eau potable et à l'assainissement. ${ }^{83}$ Il convient ici d'ouvrir une parenthèse concernant la remarque d'Amnesty International portant sur une distinction : bien que les résolutions de l'AGNU et du Conseil des droits de l'homme consacrent un droit à l'eau et à l'assainissement, certaines interprétations insistent sur l'existence des deux droits, d'une part le droit à l'eau et d'autre part le droit à l'assainissement. ${ }^{84}$ En outre, de nombreux États ne reconnaissent que le droit à l'eau mais pas le droit à l'assainissement. ${ }^{85}$

À l'heure actuelle, le droit à l'eau n'est pas consacré explicitement par une convention à vocation universelle ayant une portée générale. ${ }^{86}$ On notera avec intérêt qu'au vu des chiffres donnés par l'Organisation mondiale de la Santé (OMS) ${ }^{87}$ et du rapport du Secrétaire général, qui met en lumière que l'insalubrité de l'eau et de l'assainissement est plus mortelle que les guerres ${ }^{88}$ la reconnaissance de ces droits est cruciale.

En ce qui concerne la jurisprudence internationale en matière du droit à l'eau, on peut citer un exemple relatif aux investissements. Il s'agit de l'affaire Suez, Sociedad General de Aguas de Barcelone S.A. and Vivendi Universal S.A. v. Argentine Republic. Dans cette affaire, le mémoire des amicus curiae soulignait que l'Argentine avait des obligations relatives aux droits de l'homme, en particulier l'obligation en l'espèce d'assurer à sa population l'accès à l'eau. Selon l'Argentine, cette obligation prévalait sur ses autres obligations issues des traités bilatéraux d'investissement. Le tribunal arbitral qui ne partageait pas cette avis a conclu que l'Argentine devait respecter à la fois ses obligations relatives aux droits de l'homme et celles concernant les traités bilatéraux d'investissement. Selon le tribunal, en l'espèce, lesdites

${ }^{79}$ Caflisch, 2011, p. 389.

80 Voir http://www.fidh.org/fr/mondialisation-droits-humains/Droits-economiques-sociaux-et/Protocolefacultatif-au-PIDESC,754/les-droits-economiques-sociaux-et-culturels-desormais-justiciables-au-13231 (dernière consultation : le 27 juin 2018)

${ }^{81} \mathrm{~A} / \mathrm{RES} / 64 / 292,28$ juillet $2010, \S 1$.

${ }^{82}$ Caflisch, 2011, pp. 392-393.

${ }^{83}$ Conseil des droits de l'homme: Résolution 15/9, 30 septembre 2010; Résolution 16/2, 24 mars 2011 ; Résolution 18/1, 28 septembre 2011 et A/HRC/24/L.31, 23 septembre 2013. Pour d'autres instruments internationaux prévoyant le droit à l'eau voir Smets, 2008, pp. 8 et $\mathrm{s}$.

${ }^{84}$ Amnesty International and WASH United, 2015, p. 3.

${ }^{85}$ Idem.

${ }^{86}$ Voir Smets, 2007, pp. 30, 37 et Pellet, 2011, p. 398.

${ }^{87} \mathrm{OMS} / \mathrm{UNICEF}$, Rapport sur l'évaluation de la situation mondiale de l'approvisionnement en eau et de l'assainissement en 2000, p. 5. Voir Dupuy, 2006, p. 4.

${ }^{88}$ Rapport du Secrétaire général, Nous, les peuples : le rôle des Nations Unies au XXIe siècle, 2000, A/54/2000, p. $249 ; \S 279$. 
obligations n'étaient pas incompatibles, ni contradictoires ni mutuellement exclusives. ${ }^{89} \mathrm{Au}$ regard de cette affaire, il semble qu'il faudrait trouver une solution aux problèmes, par exemple, de concurrence et de conflit entre les normes internationales relatives aux droits de l'homme et d'autres normes, car il est important de savoir si les obligations internationales autres que celles relatives aux droits de l'homme peuvent être respectées au détriment de ces derniers. ${ }^{90}$

\section{La Protection À La Fois Régionale Et Nationale Du Droit À L'Eau}

La protection régionale et nationale du droit à l'eau témoigne d'une reconnaissance textuelle et du développement d'une protection jurisprudentielle.

Le droit à l'eau est pris en compte, explicitement ou implicitement, par des conventions, protocoles, chartes ou déclarations. Sans être exhaustif, peuvent être cites la Charte africaine des droits et du bien-être de l'enfant ; la Charte des eaux du fleuve Sénégal de 2002 (au niveau sous-régional); le Protocole additionnel à la Convention interaméricaine des droits de l'homme relatif aux droits économiques, sociaux et culturels (auquel le droit à l'eau est considéré impliqué); la Déclaration de Beppu de 2007 (en Asie-Pacifique) et la Charte européenne sur les ressources en eau de 2001 (adoptée au sein du Conseil de l'Europe) et le Protocole sur l'eau et la santé à la Convention d'Helsinki (adopté au sein de la Commission économique des Nations Unies pour l'Europe). ${ }^{91}$

Quant au niveau national, le droit à l'eau est consacré tant par des constitutions nationales, comme celles de la Slovénie, de l'Afrique du Sud, du Nicaragua, de la Bolivie, du Kazakhstan, d'Hawaï que par des législations, comme celles de la France, de l'Indonésie, du Pérou, du Cameroun..$^{92} \mathrm{Si}$ certaines constitutions le consacrent, en utilisant un langage plus «prudent $»,{ }^{93} \mathrm{~d}$ 'autres reconnaissent non seulement le droit à l'eau mais également le droit à l'assainissement, telles que la Constitution de l'Équateur et celle de l'Uruguay. ${ }^{94}$

L'importance de la reconnaissance constitutionnelle ou législative du droit à l'eau résulte, en partie, du fait qu'elle puisse influencer, entre autres, l'accès à l'eau potable pour les peuples autochtones et les migrants urbains récents; les lois et règlements relatifs à la privatisation, à l'agriculture, à la concurrence, à la pollution. ${ }^{95}$ De surcroît, sa consécration explicite le rendrait plus visible et aurait ainsi des conséquences, telles que l'obligation de l'augmentation pour les pouvoirs publics de la priorité pour l'eau; l'obligation de l'État

${ }^{89}$ Centre international pour le règlement des différends relatifs aux investissements, Suez, Sociedad General de Aguas de Barcelone S.A. and Vivendi Universal S.A. v. Argentine Republic, affaire $\mathrm{n}^{\circ}$ ARB/03/19, 30 juillet 2010, § 262. Voir Mayer, 2011, p. 181.

${ }^{90}$ Ruggie, 2011, pp. 130-131.

${ }^{91}$ Pour ces exemples cités et d'autres voir Smets, 2008, pp. 5 et s.; http://www.righttowater.info/progress-sofar/timeline-2/ et Coulée, 2011, p. 33.

Pour des instruments au niveau africain voir également les lignes directrices relatives aux rapports des États parties sur les droits économiques, sociaux et culturels dans la Charte africaine des droits de l'homme et des peuples, dites lignes directrices de Tunis de 2012 (accessible sur http://www.achpr.org/files/instruments/economic-social-cultural-

guidelines/achpr_instr_tunis_reporting_guidelines_esc_rights_2012_fra.pdf ) ainsi que la résolution de la Commission africaine des droits de l'homme et des peuples sur une approche de la gouvernance des ressources naturelles basée sur les droits de l'homme (Résolution 224, 2 mai 2012)

92 À ce sujet et pour plus d'exemples voir Bernaud, 2017, p. 320; Smets, 2008, pp. 125 et s.; Smets, 2002 b, pp. 843-844 ; Smets, 2007, pp. 127 et s. ; Brown Weiss, 2009, p. 337 et http://www.righttowater.info/progress-sofar/national-legislation-on-the-right-to-water/

${ }^{93}$ Comme celle de Zambie selon laquelle « l'État s'efforcera de faciliter un accès égal à l'eau propre et saine ». Cité par Smets, 2002b, p.844.

${ }^{94} \mathrm{http}: / /$ www.righttowater.info/progress-so-far/national-legislation-on-the-right-to-water/\#Z

${ }^{95}$ Bluemel, 2004, pp. 988 et $\mathrm{s}$. 
d'exercer le contrôle sur les services d'eau; la possibilité de disposer de l'eau d'autrui ; la justiciabilité du droit à l'eau. ${ }^{96}$ En effet, la Belgique et la Hongrie ont démontré que l'insertion du droit à l'eau dans des actes législatifs peut être efficace en cas de l'adoption des plans et des stratégies visant à mettre en œuvre ledit droit. ${ }^{97}$

À cette consécration mentionnée ci-dessus par des instruments juridiques s'ajoute le développement d'une protection jurisprudentielle. ${ }^{98}$ Dans ce sens, le droit à l'eau est reconnu par des juridictions régionales et nationales particulièrement grâce à des liens du droit à l'eau avec d'autres droits de l'homme. Ainsi, le droit d'accès à l'eau a pu être considéré dans le cadre du droit au respect de la vie privée et familiale, des droits des peuples autochtones, du droit à la santé, du droit à la propriété etc. ${ }^{99}$ Pour ne citer qu'un exemple, dans l'affaire Sufi et Elmi, l'eau limitée a été prise en compte par la Cour européenne des droits de l'homme lorsque cette dernière a conclu que l'expulsion de deux ressortissants somaliens constituerait la violation de l'article 3 portant sur l'interdiction de la torture, des peines ou traitements inhumains ou dégradants. ${ }^{100}$

Par ailleurs, les exemples suivants mettent en exergue que le droit à l'eau trouve une application concrète au sein de tribunaux nationaux. Dans l'affaire A.P. Pollution Control Board II c. Prof. M.V. Naidu and Others, la Cour suprême indienne a considéré que l'eau et l'assainissement font partie du droit à la vie. ${ }^{101}$ La Cour constitutionnelle colombienne a également proclamé le droit à l'eau. ${ }^{102}$ La Cour suprême du Botswana a conclu, dans l'affaire Mosetlhanyane and others c. Attorney General of Botswana, que les peuples autochtones ont le droit de forer des puits sur leurs terres ancestrales. ${ }^{103}$ En Argentine, des tribunaux ont adopté des décisions qui contribuent à la clarification du contenu du droit à l'eau, telles que l'affaire Menoris Comunidad Paynemil (dans laquelle le Tribunal a décidé la quantité d'eau qu'une entreprise devait fournir par jour pour la communauté autochtone) ou l'affaire Users and Consumers in Defense v. Aguas del Gran Buenos Aires S.A. (dans laquelle le juge a conclu que l'accès à l'eau devait être garanti par l'État à tous les citoyens quelles que soient leurs ressources financières). ${ }^{104}$

Dans certains pays, il s'agit d'une confirmation jurisprudentielle du droit à l'eau déjà reconnu par un acte juridique. Dans l'affaire Government of the Republic of South Africa and Others v. Grootboom and Others 2000, le tribunal de l'Afrique du Sud a appliqué le droit à l'eau et adopté une interprétation qui ressemble à celle de l'Observation $n^{\circ} 15 .{ }^{105}$ En Bolivie,

\footnotetext{
${ }^{96}$ Smets, 2007, p. 106.

${ }^{97}$ Dubreuil, 2006, p. 22.

${ }^{98}$ Pour des nombreux exemples voir WASH United and Waterlex, 2014.

${ }^{99}$ CEDH, Affaire Tătar c. Roumanie, requête $n^{\circ}$ 67021/01, 27 janvier 2009 ; CIADH, Affaire Yakye Axa Indigenous Community c. Paraguay, préc., § 167 ; Commission africaine des droits de l'homme et des peuples, 25/89-47/90-56/91-100/93 : Free Legal Assistance Group, Lawyers' Committee for Human Rights, Union Interafricaine des Droits de l'Homme, Les Témoins de Jehovah / DRC, 4 avril 1996, § 62 et 279/03-296/05: Sudan Human Rights Organisation § Centre on Housing Rights and Evictions (COHRE)/ Sudan, 27 mai 2009 , $\S 212$; CEDH, Affaire Zander c. Suède, Requête n 14282/88, 25 novembre 1993, § 27. Pour ces exemples voir Boisson de Chazournes et Tignino, 2013, § 161 ; les Règles de Berlin et Smets, 2007, p. 98.

${ }^{100}$ CEDH, Affaire Sufi et Elmi c. Royaume-Uni, requêtes n 8319/07 et 11449/07, 28 juin 2011, § 291.

${ }^{101}$ A.P. Pollution Control Board II v Prof. M.V. Naidu and Others, Civil Appeal Nos. 368-373, 1999 cité par http://www.righttowater.info/progress-so-far/national-legislation-on-the-right-to-water/\#AL

Pour les décisions d'autres juridictions voir idem.; WASH United and Waterlex, 2014 ; Dan Tarlock, 2017, § $11: 17$.

102 Cour constitutionnelle de Colombie, Décision T-546 (2009) citée par Dan Tarlock, 2017, § $11: 17$.

${ }^{103}$ Mosetlhanyane and others v. Attorney General of Botswana, Civil Appeal No. CACLB-074-10, 2011, cité par Dan Tarlock, 2017, § $11: 17$. Voir également Cour suprême de Chili, affaire Chuzmiza, 2009 cité par Cespedes, 2013, pp. 76-77.

${ }^{104}$ Bluemel, 2004, pp. 984-985.

105 Ibid., pp. 977-978.
} 
le Tribunal constitutionnel a souligné que le droit à l'eau n'est pas seulement un droit individuel mais également un droit collectif et pris une position défendant des droits des communautés indigènes. ${ }^{106}$ Ces exemples démontrent que des instances judiciaires régionales et nationales prennent en compte le droit à l'eau, et que parfois malgré l'absence dans leurs instruments juridiques d'une référence explicite à ce droit, il bénéficie d'une protection juridictionnelle.

À côté des exemples qui témoignent de la reconnaissance et de la mise en œuvre juridique du droit à l'eau, certaines jurisprudences nationales ne manquent pas d'illustrations mettant en évidence de difficultés relatives à la revendication du droit à l'eau, comme l'affaire sud-africaine Mangele c. Durban de 2002. ${ }^{107}$ Erik Bluemel identifie de nombreux défis en la matière et mentionne des questions difficiles qui attendent toujours des réponses, telles que la question du transfert de l'eau d'une région à une autre (alors que certaines lois interdisent ou limitent un tel transfert) ; celle des droits concurrentiels ou encore celle de la «tension » entre le droit à l'eau et le droit au développement. ${ }^{108}$ Par exemple, le projet de barrage du Belo Monte (Argentine) a mis en évidence que l'exploitation de l'eau comme ressource peut avoir des conséquences négatives sur le droit à l'eau des populations indigènes, ${ }^{109}$ une question donnant lieu à des mesures conservatoires prononcées par la Commission interaméricaine des droits de l'homme. ${ }^{110}$ En outre, le droit à l'eau n'est pas exempt de critiques, de questions et d'incertitudes, telles que son contenu ${ }^{111}$, la quantité d'eau qu'il implique, la priorité entre les usages de l'eau, son caractère anthropocentrique, ainsi que la place des animaux ${ }^{112}$ ou encore le droit à l'eau des États. ${ }^{113}$ Il existe donc des ambiguïtés en la matière et l'accès à l'eau continue d'être un grand problème devant l'humanité. ${ }^{114}$

\section{Conclusion}

En 2000, l'AGNU a adopté la Déclaration du Millénaire par laquelle elle décidait, en suivant le rapport du Secrétaire général, la réduction par moitié d'ici 2015 la proportion des personnes n'ayant pas d'accès à l'eau potable ou n'ayant pas de moyens de s'en procurer. ${ }^{115}$ Cet engagement a été réitéré par le Plan de mise en œuvre du Sommet mondial pour le développement durable et par "L'avenir que nous voulons» de 2012. ${ }^{116}$ Lors de la manifestation spéciale tenue le 25 septembre 2013 aux Nations Unies, le représentant du Népal soulignait le retard relatif à la mise en œuvre des programmes décennaux en raison de leur difficile adaptation aux réalités nationales. ${ }^{117} \mathrm{Au}$ cours de la même manifestation le

${ }^{106}$ Bernaud, 2017, p. 332.

107 http://www.righttowater.info/rights-in-practice/legal-approaches/legal-approach-case-studies/challenges-inclaiming-the-right-to-water-south-africa/

108 Bluemel, 2004, p. 987.

109 Higuet, 2012.

${ }^{110}$ Idem.

${ }^{111}$ CIADH, PM 382/10- Indigenous Communities of Xingu River Basin, Pará, Brazil, 2011. Voir Higuet, 2012.

112 Pour ces questions voir Brown Weiss, 2009, p.333 ; Lynch, 2011, pp. 411-412 ; Smets, 2011, p. 356 et Smets, 2007 , pp. 20 et s.

${ }^{113}$ Concernant le droit à l'eau des États, en 2010, l'Éthiopie, l'Ouganda, le Rwanda et la Tanzanie ont conclu l'Accord-cadre sur la coopération dans le bassin du fleuve Nil. En 2011, le Burundi a rejoint ces États. Cependant l'Égypte qui ne soutient pas cet accord, a prétendu qu'il avait affecté « son "droit à l'eau"». Coulée, 2011, p.14.

${ }^{114}$ Voir Coulée, 2011, p.38 et Dupuy, 2006, pp. 10-11.

115 Déclaration du Millénaire, A/RES/55/2, 8 septembre 2000, § 19.

${ }^{116}$ Rapport du Sommet mondial pour le développement durable, Johannesburg Afrique du Sud, 26 août-4 septembre 2002, A/CONF.199/20, pp. 9 et s., $\S \S 7$ et s. et L'avenir que nous voulons, $\S \S 119$ et s. Voir également le projet de résolution sur «Document final de la manifestation spéciale consacrée au bilan de l'action entreprise pour atteindre les objectifs du Millénaire pour le développement », A/68/L.4, $1^{\text {er }}$ octobre $2013, \S 8$.

117 https://www.un.org/press/fr/2013/AG11440.doc.htm (dernière consultation : le 27 juin 2018). 
représentant de la Mongolie précisait que pour son pays « la priorité [était] l'accès à l'eau » et la nécessité d'une convention des Nations Unies sur la gestion des ressources en eau. ${ }^{118}$ Quinze ans après l'adoption de la Déclaration du Millénaire, en 2015 les Objectifs de développement durable ont adoptés. Son objectif 6 est de «[g]arantir l'accès de tous à des services d'alimentation en eau et d'assainissement gérés de façon durable ${ }^{119}{ }^{19} \mathrm{En}$ effet, les nouvelles données relatives à la situation mondiale en matière d'accès à l'eau sont très inquiétantes. ${ }^{120}$ Tout cela démontre par conséquent que ce problème attend toujours une solution.

Le développement normatif concernant la protection du droit d'accès à l'eau ainsi que la protection des ressources en eau demeure limité dans le sens où certains problèmes restent d'actualité et, pour l'instant, sans réponse juridique d'une part et d'autre part, la compréhension de cette problématique requiert un changement de paradigme. ${ }^{121}$ Finalement, il s'avère indispensable de constater qu'actuellement la protection juridique de l'eau n'est ni facile ni suffisante. ${ }^{122}$

\section{Bibliographie}

Livres, articles et Recueil des Cours de l'Académie de droit international de La Haye

Arcari, M. (2012). Les interactions entre le régionalisme et l'universalisme dans le droit international des ressources en eau. In S. Doumbé-Billé (sous la coordination), La régionalisation du droit international, 347-360. Bruxelles : Bruylant.

Bernaud, V. (2017). La Consécration d'un droit fondamental à l'eau est possible. Etude de droit comparé le démontrant. Revue française de droit constitutionnel, 317-342.

Bluemel, E. B. (2004). The Implications of Formulating a Human Right to Water. Ecology Law Quarterly, 957-1006.

Boisson de Chazournes, L. et Tignino, M. (2013). Droit international et eau douce. JurisClasseur, Fasc. 2900.

Brown Weiss, E. (2009). The Evolution of International Water Law. Recueil des Cours de l'Académie de droit international de La Haye (RCADI), 163-404.

Caflisch, L. (2011). Le droit à l'eau- Un droit de l'homme internationalement protégé ?. In SFDI, L'eau en droit international, 385-394. Paris : Pedone.

Cardenas, Y. V. (2007). La Construction sociale du statut juridique de l'eau en Amérique du Nord. Lex Electronica, 1-17, accessible sur http://www.lexelectronica.org/files/sites/103/12-2_vega-cardenas.pdf (dernière consultation : 27 juin 2018).

Cespedes, R. (2013). Indigenous People's Human Rights to a Clean Environment, Environmental Impact Assessment and ILO-Convention 1969. Warwick Student Law Review, 71-80.

Coulée, F. (2011). Rapport général. Du droit international de l'eau à la reconnaissance internationale d'un droit à l'eau. In SFDI, L'eau en droit international, 9-40. Paris : Pedone.

Cuq, M. (2013). L'eau en droit international. Convergences et divergences dans les approches juridiques. Bruxelles, Larcier.

Dan Tarlock, A. (2017). Law of Water Rights and Resources. Chapter 11 : International Water Allocation. (Accès depuis Westlaw).

\footnotetext{
118 Idem.

119 A/RES/70/1, 25 septembre 2015, pp. 15 et 20.

120 « 844 million lack basic water services, 2.1 billion lack managed drinking water, 4.5 billion lack access to safely managed sanitation and 892 million still practice open defecation. Only 27 per cent of the population in least developed countries has access to soap and water for handwashing on premises ». UN Water, 2018.

${ }^{121}$ Paquerot, 2002, p. 88 et 78-83.

${ }^{122}$ Doumbé-Billé, 1994, p. 202.
} 
Doumbé-Billé, S. (1994). L'Agenda 21 et les eaux douces. In M. Prieur et S. Doumbé-Billé (dir.), Droit de l'environnement et développement durable, 196-210, Limoges: PLUM.

Dubreuil, C. (sous la direction de P. Van Hofwegen). Le droit à l'eau : Du concept à la mise en ouvre, Conseil mondial de l'eau.

Dupuy, P.-M. (2006). Le droit à l'eau, un droit international ?. EUI Working Papers Law, 112 accessible

http://cadmus.eui.eu/bitstream/handle/1814/4252/LAW\%202006.6\%20Dupuy.pdf (dernière consultation : 27 juin 2018).

Higuet, F. (2012). Le barrage de Belo Monte : un conflit majeur entre la gestion de l'eau comme ressource et le droit à l'eau des communautés indigènes. Accessible sur http://www.rampedre.net/jurisprudence/drinkable_water/br\%C3\%A9sil_\%C3\%A9tat_ du_par\%C3\%A1_le_barrage_de_belo_monte\#conclusion (dernière consultation : 25 juin 2018).

Kohen, M. G. (2011). Les principes généraux du droit international de l'eau à la lumière de la jurisprudence récente de la Cour internationale de justice. In SFDI, L'eau en droit international, 91-108. Paris : Pedone.

Lavieille, J.-M. (2010). Droit international de l'environnement. Paris : Ellipses.

Lawson, F. (2013). Sustainable Development along International Watercourses: Is progress being made?, University of Denver Water law Review, 323-348.

Lynch, R. (2011). Book Note: Karen Bakker, Privatizing Water: Governance Failure and the World Water Crisis, Cornell University Press, New York (2010); 303 pp. University Denver Water Law Review, 411-412.

Mayer, P. (2011). Les arbitrages CIRDI en matière d'eau. In SFDI, L'eau en droit international, 163-183, Paris : Pedone.

Ngyuen, Q. D., Daillier, P., Forteau, M. et Pellet, A. (2009). Droit international public. Paris : LGDJ.

Paquerot, S. (2002). Le statut des ressources vitales en droit international : essai sur le concept de patrimoine commun de l'humanité. Bruxelles : Bruylant.

Paquerot, S. (2011). Les développements normatifs du droit international de l'eau: contradictions non résolues et ambivalence normative. In SFDI, L'eau en droit international, 71-90 : Paris : Pedone.

Pellet, A. (2011). Conclusions générales. In SFDI, L'eau en droit international, 395-402 : Paris : Pedone.

Ruggie, J. G. (2011). The Construction of the UN "protect, respect and remedy" framework for business and human rights: confessions of a principled pragmatist. European Human Rights Law Review, 127-133.

Smets, H. (2002a). Le droit à l'eau. Conseil européen du droit de l'environnement, 1-111, accessible

sur http://dev.worldwatercouncil.org/fileadmin/wwc/Programs/Right_to_Water/Pdf_doct/ eau_CEDE_20021.pdf (dernière consultation : 28 juin 2018).

Smets, H. (2002b). Reconnaissance et mise en œuvre du droit à l'eau. Revue trimestrielle des droits de l'homme, 837-852.

Smets, H. (2007). La reconnaissance officielle du droit à l'eau en France et à l'international. Agence Française de Développement. Département de la Recherche, 1-168.

Smets, H. (2008). L'accès à l'eau potable et le droit international. Académie de l'Eau, pp. 150, accessible sur http://www.aihrresourcescenter.org/administrator/upload/documents/pot.pdf (dernière consultation 28 juin 2018). 
Smets, H. (2011). La reconnaissance internationale de priorités dans les usages de l'eau. In SFDI, L'eau en droit international, 349-365. Paris : Pedone.

Sohnle, J. (2002). Le droit international des ressources en eau douce : solidarité contre souveraineté. Paris : La Documentation française.

Sohnle, J. (2011). Les spécificités de la protection internationale de l'environnement en matière de ressources en eau- que faire pour qu'un poisson soit heureux comme un poisson dans l'eau (douce) ?, In SFDI, L'eau en droit international, 305-320. Paris : Pedone.

\section{Jurisprudence}

\section{Cour permanente de Justice internationale}

CPJI, Affaire relative à la juridiction territoriale de la Commission internationale de l'Oder, 10 septembre 1929, Série A, nº 23.

\section{Cour internationale de Justice}

CIJ, Affaire relative au projet Gabčikovo-Nagymaros, arrêt du 25 septembre 1997, CIJ Rec. 1997, p. 7.

CIJ, Affaire relative à des Usines de pâte à papier sur le fleuve Uruguay (Argentine c. Uruguay), arrêt du 20 avril 2010, CIJ Rec. 2010, p. 14.

CIJ, Construction d'une route au Costa Rica le long du fleuve San Juan (Nicaragua c. Costa Rica), Requête introductive d'instance du 22 décembre 2011.

Commission africaine des droits de l'homme et des peuples

Commission africaine des droits de l'homme et des peuples, 25/89-47/90-56/91-100/93 : Free Legal Assistance Group, Lawyers' Committee for Human Rights, Union Interafricaine des Droits de l'Homme, Les Témoins de Jehovah / DRC, 4 avril 1996.

Commission africaine des droits de l'homme et des peuples, 279/03-296/05 : Sudan Human Rights Organisation \& Centre on Housing Rights and Evictions (COHRE)/ Sudan, 27 mai 2009.

\section{Cour européenne des droits de l'homme}

CEDH, Affaire Zander c. Suède, Requête n 14282/88, 25 novembre 1993.

CEDH, Affaire Tătar c. Roumanie, requête n 67021/01, 27 janvier 2009.

CEDH, Affaire Sufi et Elmi c. Royaume-Uni, requêtes n 8319/07 et 11449/07, 28 juin 2011.

Cour interaméricaine des droits de l'homme

CIADH, Case of the Yakye Axa Indigenous Community v. Paraguay, Affaire Yakye Axa Indigenous Community c. Paraguay, 17 juin 2005.

CIADH, Xákmok Kásek Indigenous Community v Paraguay, 24 August 2010.

CIADH, PM 382/10- Indigenous Communities of Xingu River Basin, Pará, Brazil, 2011.

\section{Sentences arbitrales}

RSA, Affaire du lac Lanoux -Espagne, France-, 16 novembre1957, vol. XII, pp. 281-317.

Centre international pour le règlement des différends relatifs aux investissements, Suez, Sociedad General de Aguas de Barcelone S.A. and Vivendi Universal S.A. v. Argentine Republic, affaire $\mathrm{n}^{\circ} \mathrm{ARB} / 03 / 19,30$ juillet 2010 .

\section{Documents officiels}

AGNU, Rés. 2669 (XXV), 8 décembre 1970

A/RES/54/175, 15 février 2000

A/RES/55/2, 8 septembre 2000

A/RES/61/295, 13 septembre 2007

A/RES/64/292, 28 juillet 2010

A/RES/66/288, 27 juillet 2012

A/RES/70/1, 25 septembre 2015

A/CN.4/244/Rev.1

A/CONF.199/20, 2002 
A/68/L.4, $1^{\text {er }}$ octobre 2013

A/HRC/24/L.31, 23 septembre 2013

A/64/692, 4 mars 2010

Amnesty International and WASH United, Recognition of the human rights to water and sanitation by UN Member States at the international level, 2015, p. 3, accessible sur https://www.amnesty.org/fr/documents/ior40/1380/2015/en/

Association du droit international, Règles de Berlin sur les ressources en eau, 2004.

CDI, Projet d'articles sur le droit des aquifères transfrontières, in Annuaire de la Commission du droit international, 2008, Vol. II (Deuxième partie).

Commission africaine des droits de l'homme et des peuples sur une approche de la gouvernance des ressources naturelles basée sur les droits de l'homme, Résolution 224, 2 mai 2012.

Comité des droits économiques, sociaux et culturels, Rapport sur les vingt-huitième et vingtneuvième sessions (29 avril-17 mai 2002, 11_ 29 avril 2002), Annexe IV, Observation générale $n^{\circ} 15$ (2002), Le droit à l'eau (art.11 et 12 du Pacte).

Conseil de l'Union européenne, «Protection des ressources en eau et gestion durable intégrée de l'eau dans l'Union européenne et au-delà -Conclusions du Conseil- », $3013^{\text {ème }}$ session du Conseil Environnement, 21 juin 2011.

Conseil des droits de l'homme, Résolution 15/9, 30 septembre 2010

Conseil des droits de l'homme, Résolution 16/2, 24 mars 2011

Conseil des droits de l'homme, Résolution 18/1, 28 septembre 2011

ECE/MP.WAT/14, 12 janvier 2004OMS/ UNICEF, Rapport sur l'évaluation de la situation mondiale de l'approvisionnement en eau et de l'assainissement en 2000, accessible sur http://www.who.int/water_sanitation_health/monitoring/gwssa2000f.pdf?ua=1 (dernière consultation : 27 juin 2018).

PNUE, Décision 27/3 : Directives internationales sur la qualité de l'eau pour les écosystèmes, UNEP/GC.27/17, $27^{\text {ème }}$ session du Conseil d'administration/ Forum ministériel mondial sur l'environnement, 18-22 février 2013.

Rapport de la Conférence internationale sur la population et le développement, Le Caire, 5-13 septembre 1994, A/CONF.171/13/Rev.1.

Rapport du Secrétaire général, Nous, les peuples : le rôle des Nations Unies au XXIe siècle, 2000, A/54/2000.

UNEP, 2002, Atlas of International Freshwater Agreements, accessible sur http://www.unep.org/publications/search/pub_details_s.asp?ID=115

UNESCO, Les solutions fondées sur la nature pour la gestion de l'eau, Rapport mondial des Nations Unies sur la mise en valeur des ressources en eau 2018, 2018, accessible sur http://unesdoc.unesco.org/images/0026/002614/261466f.pdf(dernière onsultation : 25 juin 2018).

UN Water, Highlights [from SGD 6 Synthesis Report 2018 on Water and Sanitation], 2018, accessible sur http://dialogue.unwater.org/resources/ (dernière consultation: 25 juin 2018).

WASH United and Waterlex, The Human Rights to Water and Sanitation in Courts Worldwide. A selection of Nations, Regional and International Case Law, 2014.

\section{Sites d'Internet}

http://treaties.un.org/

http://www.fidh.org/

http://www.righttowater.info/

http://www.achpr.org/

https://www.un.org/ 